\title{
Ependymal Tumor
}

National Cancer Institute

\section{Source}

National Cancer Institute. Ependymal Tumor. NCI Thesaurus. Code C6770.

A group of neoplasms which arise from the ependymal lining of the cerebral ventricles and from the remnants of the central canal of the spinal cord. Ependymal tumors occur predominantly in children and young adults with varied morphological features and biological behavior. There are 4 types: ependymoma, anaplastic ependymoma, myxopapillary ependymoma and subependymoma. (WHO) 